\title{
HEAT TRANSFER FROM THERMAL PLASMAS TO NEIGHBORING WALLS OR ELECTRODES
}

\author{
E. PFENDER \\ Heat Transfer Division, Department of Mechanical Engineering, University of Minnesota, \\ Minneapolis, MN 55455, USA
}

\begin{abstract}
A successful exploitation of thermal plasmas in the field of high temperature chemistry and material processing requires a fundamental understanding of the interaction between a plasma and matter in the condensed phase. In this paper some of the basic aspects involved in the heat exchange between a thermal plasma and solids or liquids are reviewed, with emphasis on recent experimental results obtained at the University of Minnesota. These results refer to plasma heat transfer situations with and without electric current flow to or from the surface under consideration. For both situations methods have been developed which allow fairly accurate measurements of local heat fluxes over a wide range of plasma parameters.
\end{abstract}

\section{INTRODUCTION}

A plasma, sometimes called the fourth state of matter offers a number of unusual features with intriguing possibilities for applications. The high energy content of a plasma represents one of its outstanding features which holds promise for new developments in the field of high temperature chemistry and material processing. Our present energy situation demands an extensive exploration of this potential.

An effective utilization and exploitation of the plasma state requires a thorough understanding of the heat transfer process from a plasma to a solid or a liquid. This process is much more complicated than in the case of an ordinary gas. The existence of free electrons and positive ions in a plasma and the steep gradients of the plasma parameters, particularly in the vicinity of walls or electrodes, give rise to a number of effects which are still poorly understood. The interaction with relatively strong radiation fields which are typical for thermal plasmas, complicates the situation further.

Research in the field of plasma heat transfer received a strong impetus by plasma applications related to space flight and reentry simulation. Relevant literature references are summarized in Ref. 1.

There seems to be renewed interest in this field, precipitated by the energy crisis. Both the availability of nuclear fuels and the abundant supplies of coal suggest that an Electric Energy Economy may be at least part of the solution to the long term energy problems. A shift to electricity as the major energy base will have a profound impact on high temperature chemistry and high temperature material processing for which plasma processes may find increasing applications. ${ }^{2-21}$

In this paper, recent results of experimental and analytical plasma heat transfer studies, conducted in the High Temperature Laboratory, Heat Transfer Division, University of Minnesota will be summarized. No attempt will be made to give a comprehensive literature survey. Only a few publications will be mentioned which are directly related to the material covered in this paper.

In the experimental work electric arcs have been used as a convenient means for generating plasmas in a temperature range from approximately $8 \times 10^{3}$ to $3 \times 10^{4} \mathrm{~K}$ and electron densities ranging from $10^{16}$ to $10^{18} \mathrm{~cm}^{-3}$. These plasmas can be described as continua since most of the studies refer to atmospheric pressure and the temperature is usually high enough so that the plasmas may be considered as thermal plasmas, i.e. they approach a state of Local Thermodynamic Equilibrium (LTE). The cooling effect caused by walls and electrodes bordering the plasma or by particulate matter injected into the plasma extends, generally, into the plasma in a thin layer only which can, in the usual terminology be described as a boundary layer.

Analytical investigations in plasma heat transfer are almost entirely restricted to laminar flow because the degree and the nature of turbulence in the plasma flow is only poorly known.

The paper is divided into three sections. The first section reviews some basic aspects of plasma heat transfer, including a brief discussion of the various mechanisms which may contribute to the total heat flow. In the second section, results of heat transfer studies to surfaces will be reviewed assuming that these surfaces are not subject to appreciable current flow. This situation prevails in all kinds of plasma generating devices in which the plasma is in "contact" with walls or other structural elements of the device (excluding electrodes) as well as in plasma applications in which materials are exposed to or forced into the hot plasma.

If there is a substantial flow of electric current to a surface, heat transfer may be dominated by the current flow. This situation will be discussed in the last section which deals with electrode heat transfer, in particular with heat transfer to the anode in high intensity arcs.

\section{BASIC ASPECTS OF PLASMA HEAT TRANSFER}

For a proper assessment of plasma heat transfer it is useful to evaluate qualitatively how much heat transfer in a plasma is expected to differ from heat transfer in an ordinary gas at low temperature.

\section{(A) Qualitative considerations}

The heat transfer phenomenon and, in particular, the thermal boundary layer are well understood for a cool, solid body immersed in a laminar hot gas stream in which no chemical reactions occur. In this simple situation, the heat transfer can be predicted by a dimensionless parameter, the Nusselt number

$$
\mathrm{Nu}=f(\operatorname{Pr}, \mathrm{Re})
$$

(Pr $=$ Prandtl number, $\operatorname{Re}=$ Reynolds number $).$ With 
some modifications, similar relationships hold for dissociating gases, provided that the Lewis number which describes the diffusion of the species is close to unity.

If one now considers heat transfer in a temperature range high enough for ionization to occur, one might be tempted to expect a strong increase of the heat transfer coefficient because the free electrons contribute strongly to the thermal conductivity as they do in a metal. But this is not the case-at least not on a cold catalytic wall-because its surface is always separated by a cool less ionized layer from the hotter part of the boundary layer and from the main stream. This layer will be thicker when chemical equilibrium exists than for a chemically frozen boundary layer. Such a layer acts as a thermal insulator and prevents a large increase in heat transfer. From a purely theoretical point of view, only on a completely non-catalytic surface an electron density with finite values would exist throughout the boundary layer and considering electronic conduction only, a strong increase of the heat transfer to the surface would have to be expected.

There is another factor involved in heat transfer from a plasma which tends to reduce the heat flux. In the interdiffusion of electrically charged and uncharged particles in an ionized gas, the electrons and ions move as if they were joined together-(ambipolar diffusion). The diffusion coefficient $D$ for this process is approximately a factor of two larger than the diffusion coefficient for the corresponding neutral particles. It therefore maintains its order of magnitude in the Lewis number

$$
\mathrm{Le}=\frac{\rho c_{p} D}{k}
$$

( $\rho=$ density, $\quad c_{p}=$ specific heat at constant pressure, $D=$ diffusion coefficient, $k=$ thermal conductivity). The thermal conductivity in a plasma, however, increases possibly by an order of magnitude or even more. This causes the Lewis number to assume values smaller than one. Therefore, a correction term has to be applied to the heat transfer coefficient which reduces the Nusselt number. ${ }^{1}$

These considerations demonstrate that the Nusselt number and the heat flux to the surface are not as strongly influenced by the presence of electrons as one would expect and that the heat flux in a boundary layer at composition equilibrium will be smaller than in a frozen boundary layer. Experiments which verify these conclusions will be discussed in later paragraphs.

Another mechanism which is of minor importance in an ordinary gas at low temperature may play an important role if plasmas are considered. A thermal plasma is a strongly radiating gas. In general, the integrated radiation intensity is a function of temperature and pressure for a given plasma. For the temperature range which is of interest in this review, the radiation intensity increases strongly with temperature and also with pressure. For extremely high pressures $(>100 \mathrm{~atm})$ the radiation intensity approaches that of a blackbody.

Unfortunately, there is no general description of radiative heat transfer from a plasma to a surface because this transfer depends strongly on the nature of the plasma and on the entire configuration. Only in extremely simple situations of optically thin plasmas, generated from pure, monatomic gases, reasonably accurate values of the emission coefficient may be available for a prediction of radiative heat transfer.
In applications which utilize uncooled electrodes (for example carbon or graphite) radiative heat transfer from the electrodes to the material to be heated by the plasma may play an important role in the overall energy balance.

\section{(B) Transport equations}

In this section a plasma will be considered which is macroscopically at rest. Such a plasma consists, in general, of molecules, atoms, ions and electrons, each again possibly comprising several species. Diffusion processes will set in when the concentration of the components varies locally. The analysis of this diffusion process is extremely involved and simplifications are, therefore, usually introduced. Such simplifications have been discussed, for instance, in a review by Chung. ${ }^{22}$ For the following considerations the "binary diffusion model" will be utilized. This model replaces, with regard to diffusion, the actual plasma by a two-component mixture, one component comprised of the molecules and the other of the atoms, ions and electrons. The justification for the use of such a model is the fact that at sufficiently high densities Coulomb forces prevent the ions and electrons from being separated by a diffusion process. The atoms and ions, on the other hand, differ less in their molecular weight than both differ from the molecular weight of the molecules. The binary diffusion model for this reason was found to give useful results and will be used in the basic equations describing mass and heat fluxes.

A diffusional mass flux can be generated by a concentration gradient, by a pressure gradient, by a temperature gradient and by body forces (for example electric fields). In a boundary layer, diffusion occurs in a direction in which pressure gradients are negligibly small. For this reason pressure diffusion will be disregarded. Diffusion caused by an electric field tends to separate the electrons and the ions. Coulomb forces acting between the particles themselves, however, counteract this tendency and diffusion by this type of body forces will, therefore, be neglected for the plasmas considered in this paper. Diffusion due to gravitational forces is also negligible. The remaining diffusion by concentration gradients and by temperature gradients is described in the two-component gas mixture by the following equation ${ }^{23}$

$$
j_{D 1}=-\rho D\left[\frac{\partial w_{1}}{\partial y}+w_{1} w_{2} \alpha \frac{\partial}{\partial y}(\ln T)\right] .
$$

In this equation it is assumed that a concentration and temperature gradient exist in $y$-direction. The symbol $j_{D 1}$ denotes the mass flux per unit time and area of component one in this direction, $w_{1}$ is the mass fraction of component one, $w_{2}$ the mass fraction of component two, $T$ denotes the temperature, $\rho$ the density of the mixture, $D$ the binary mass diffusion coefficient, and $\alpha$ the thermal diffusion ratio. The mass flux $j_{D 2}$ of component 2 is by definition equal to $-j_{D 1}$. The corresponding heat flux $q$ (per unit time and area) is described by the following equation

$$
q=-k \frac{\partial T}{\partial y}+\left(i_{1}-i_{2}\right) j_{D 1}-\alpha R T \frac{M^{2}}{M_{1} M_{2}} j_{D 1} .
$$

The first term in this equation describes energy transport by heat conduction. The second term gives the energy transport by enthalpy interdiffusion and the third term describes energy transport by the diffusion thermo effect. The symbol $k$ denotes the thermal conductivity, $i_{1}$ and $i_{2}$ 
are enthalpies of component 1 and component 2, respectively, $R$ is the gas constant, and $M$ the molecular weight of the mixture. $M_{1}$ and $M_{2}$ denote the molecular weights of the two components, respectively. Equation (4) can also be written in a different way by introducing the mass flux given by eqn (3).

$$
\begin{aligned}
& q=-\left[k+\frac{\rho D w_{1} w_{2} \alpha}{T}\right.\left.\left(i_{1}-i_{2}\right)-\rho D \frac{w_{1} w_{2} \alpha^{2} R M^{2}}{M_{1} M_{2}}\right] \frac{\partial T}{\partial y} \\
&-\rho D\left[\left(i_{1}-i_{2}\right)-\alpha R T \frac{M^{2}}{M_{1} M_{2}}\right] \frac{\partial w_{1}}{\partial y}
\end{aligned}
$$

The two eqns (4) and (5) allow different interpretations of the thermal conductivity for a two-component mixture. The transport property $k$ used in eqn (4) describes the energy transport under the condition that the mass flux $j_{D 1}$ is zero. One can, however, also interpret the term in the bracket of eqn (5) which is multiplied by the temperature gradient as a thermal conductivity describing the energy transport under the condition that no concentration gradient exists.

Frequently, the contribution of thermal diffusion and of the diffusion thermo effect is relatively small so that in a first approximation eqns (3) and (4) or (5) simplify to

$$
\begin{gathered}
j_{D 1}=-\rho D \frac{\partial w_{1}}{\partial y} \\
q=-k_{f} \frac{\partial T}{\partial y}-\left(i_{1}-i_{2}\right) \rho D \frac{\partial w_{1}}{\partial y}
\end{gathered}
$$

The difference between the two conductivities discussed above has now disappeared. However, an index $f$ is added to the symbol $k$, indicating that the first term on the right-hand side of eqn (7) considers energy transport due to heat conduction only, and does not include the energy transport by enthalpy interdiffusion described by the second term of the equation.

The heat flux $q$ may be written differently, replacing the temperature $T$ in eqn (7) by the enthalpy $i$ of the mixture. ${ }^{1}$ With this replacement eqn (7) reads

$$
q=-\frac{k_{f}}{c_{p f}} \frac{\partial i}{\partial y}-\rho D\left(1-\frac{1}{\mathrm{Le}_{f}}\right)\left(i_{1}-i_{2}\right) \frac{\partial w_{1}}{\partial y} .
$$

The Lewis number in this equation is defined as

$$
\mathrm{Le}_{f}=\frac{\rho c_{p f} D}{k_{f}}
$$

Indices $f$ are again added to the thermal conductivity and to the specific heat, indicating that they describe the energy transport and the enthalpy, respectively, measured while the concentration is held constant. They are referred to as "frozen properties." The last equation can also be written as

$$
q=-\frac{\mu}{\operatorname{Pr}_{f}} \frac{\partial i}{\partial y}-\rho D\left(1-\frac{1}{\mathrm{Le}_{f}}\right)\left(i_{1}-i_{2}\right) \frac{\partial w_{1}}{\partial y}
$$

with the frozen Prandtl number $\operatorname{Pr}_{f}=c_{p f} \mu / k_{f}$. This equation is of advantage because in many cases the Lewis number of a binary mixture differs but little from the value one. An. analysis based on the assumption of a Lewis number equal to one serves then as a good

$\dagger$ This assumption is made in many analyses of reentry heat transfer. approximation to actual conditions. For such a mixture, the second term in eqn (10) is equal to zero, simplifying the equation for the energy flux considerably.

If local chemical equilibrium between the various species within the boundary layer exists or is closely approached, $\dagger$ the local mass fraction (at a prescribed pressure) is only a function of the temperature and consequently of the enthalpy. Therefore eqn (10) can then be written in the following form

$$
q=-\left[\frac{\mu}{\operatorname{Pr}_{f}}+\rho D\left(1-\frac{1}{\mathrm{Le}_{f}}\right) \frac{\partial w_{1}}{\partial i}\left(i_{2}-i_{2}\right)\right] \frac{\partial i}{\partial y} .
$$

The whole term within the brackets is now a parameter, depending on the local state in the plasma, and can be used to define a Prandtl number, $\mathrm{Pr}_{e}$, according to the equation

$$
q=-\frac{\mu}{\operatorname{Pr}_{\mathrm{e}}} \frac{\partial i}{\partial y}
$$

This equation now describes, together with the equilibrium Prandtl number, the heat flux in a plasma under the condition of local chemical equilibrium and is useful in heat transfer calculations which are based on that condition. A comparison of eqns (10) and (12) shows that the difference between the frozen Prandtl number and the equilibrium Prandtl number vanishes for a gas with a Lewis number equal to one.

The equations for the mass and energy flux derived in this paragraph will now be utilized to obtain the boundary layer equations for a two-component gas mixture.

\section{(C) Laminar boundary layer equations}

The equations describing conservation of mass, species, momentum, and energy for plane and rotationally symmetric, laminar boundary layers of a two-component gas mixture can be written in the following form

$$
\begin{gathered}
\frac{\partial}{\partial x}\left(\rho r^{n} u\right)+\frac{\partial}{\partial y}\left(\rho r^{n} v\right)=0 \\
\rho u \frac{\partial w_{1}}{\partial x}+\rho v \frac{\partial w_{1}}{\partial y}=-\frac{\partial}{\partial y} j_{D_{1}}+\kappa_{1} \\
\rho u \frac{\partial u}{\partial x}+\rho v \frac{\partial u}{\partial y}=-\frac{\mathrm{d} p}{\mathrm{~d} x}+\frac{\partial}{\partial y}\left(\mu \frac{\partial u}{\partial y}\right)
\end{gathered}
$$

$$
\begin{aligned}
\rho c_{p}\left(u \frac{\partial T}{\partial x}+v \frac{\partial T}{\partial y}\right)-u \frac{\mathrm{d} p}{\mathrm{~d} x} & =-\frac{\partial q}{\partial y}+\mu\left(\frac{\partial u}{\partial y}\right)^{2} \\
& -\left(i_{1}-i_{2}\right)\left[\kappa_{1}+\frac{\partial}{\partial y}\left(\rho D \frac{\partial w_{1}}{\partial y}\right)\right]
\end{aligned}
$$

The coordinates $x$ and $y$ as well as the length $r$ are explained in Fig. 1. The exponent $n$ is zero for plane flow and equal to one for rotationally symmetric flow. The velocity components $u$ and $v$ are also indicated in Fig. 1 . Parameters without an index refer to the mixture. The index one refers to component one. The symbol $\kappa_{1}$, in eqns (14) and (16) denotes the rate of mass generation of component one per unit volume and time by a chemical reaction. The mass flow rate $j_{D 1}$ introduced into eqn (14) yields

$$
\rho u \frac{\partial w_{1}}{\partial x}+\rho v \frac{\partial w_{1}}{\partial y}=\frac{\partial}{\partial y}\left(\rho D \frac{\partial w_{1}}{\partial y}\right)+\kappa_{1} .
$$

The energy equation can be written in two different ways 


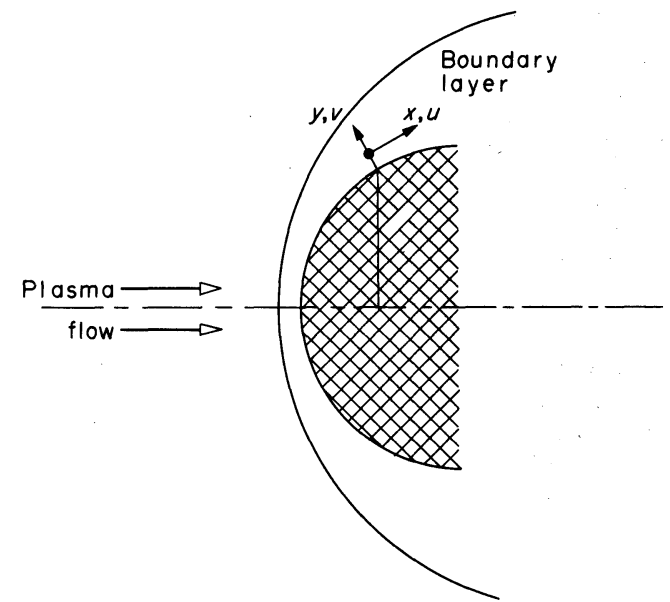

Fig. 1. Coordinates for a rotationally symmetric boundary layer.

depending on which of the two eqns (7) or (11) is introduced into eqn (16)

$$
\begin{aligned}
\rho u \frac{\partial i^{0}}{\partial x}+\rho v \frac{\partial i^{0}}{\partial y}= & \frac{\partial}{\partial y}\left(\frac{\mu}{\operatorname{Pr}_{f}} \frac{\partial i^{0}}{\partial y}\right)+\frac{\partial}{\partial y}\left[\left(1-\frac{1}{\operatorname{Pr}_{f}}\right) \mu \frac{\partial}{\partial y}\left(\frac{u^{2}}{2}\right)\right] \\
& +\frac{\partial}{\partial y}\left[\rho D\left(1-\frac{1}{\operatorname{Le}_{f}}\right)\left(i_{1}-i_{2}\right) \frac{\partial w_{1}}{\partial y}\right] \\
\rho c_{p}\left(u \frac{\partial T}{\partial x}+v \frac{\partial T}{\partial y}\right) & -u \frac{\mathrm{d} p}{\mathrm{~d} x}=\frac{\partial}{\partial y}\left(k_{f} \frac{\partial T}{\partial y}\right)+\mu\left(\frac{\partial u}{\partial y}\right)^{2} \\
& +\rho D\left(c_{p 1}-c_{p 2}\right) \frac{\partial T}{\partial y} \frac{\partial w_{1}}{\partial y}-\left(i_{1}-i_{2}\right) \kappa_{1}
\end{aligned}
$$

The symbol $i^{0}$ in eqn (18a) indicates the total enthalpy which includes kinetic energy. One of these equations is used in analyzing heat transfer processes in a plasma which is not in composition equilibrium.

For equilibrium, the energy equations can again be simplified to

$$
\begin{aligned}
& \rho u \frac{\partial i^{0}}{\partial x}+\rho v \frac{\partial i^{0}}{\partial y}=\frac{\partial}{\partial y}\left(\frac{\mu}{\operatorname{Pr}_{\mathrm{e}}} \frac{\partial i^{0}}{\partial y}\right)+\frac{\partial}{\partial y}\left[\left(1-\frac{1}{\mathrm{Pr}_{\mathrm{e}}}\right) \mu \frac{\partial}{\partial y}\left(\frac{u^{2}}{2}\right)\right] \\
& \rho c_{p}\left(u \frac{\partial T}{\partial x}+v \frac{\partial T}{\partial y}\right)-u \frac{\mathrm{d} p}{\mathrm{~d} x}=\frac{\partial}{\partial y}\left(k_{\mathrm{e}} \frac{\partial T}{\partial y}\right)+\mu\left(\frac{\partial u}{\partial y}\right)^{2} .
\end{aligned}
$$

These equations have to be further modified in the presence of magnetic fields and when electrons enter or leave the surface on which the boundary layer borders.

If radiation is important, a corresponding term has to be added to the energy equation. As previously mentioned, radiative heat transfer is difficult to describe in terms which are valid for every conceivable situation.

Boundary conditions have to be specified at the wall surface and at the outer edge of the boundary layer. At the outer edge it is customary to use the free stream conditions of the plasma. At the wall surface, both velocity components are zero if evaporation and ablation of wall material is excluded.

It should be pointed out that heat transfer under the conditions as outlined above can be predicted to a moderate degree of accuracy only, because a description of the heat transfer process requires the knowledge of thermodynamic and transport properties of the plasma. Since the thermodynamic state of a plasma, especially near a bordering surface when significant deviations from LTE may occur, is often not accurately known, the available thermodynamic and transport properties of plasmas which are based on the existence of LTE are no longer of much help. Furthermore, the available equilibrium plasma transport properties at higher temperature levels are still subject to uncertainties. This uncertainty influences also any analytical work which attempts to describe various heat transfer situations.

\section{HEAT TRANSFER TO A SURFACE WITHOUT ELECTRIC CURRENT FLOW}

In this section two experimental approaches will be discussed for determining local heat fluxes from plasmas to walls which are not subject to an appreciable current flow. The theoretical basis for this situation has been reviewed in section II.

One of the first systematic experimental investigations of plasma-wall heat transfer has been reported by Mason ${ }^{24}$ who utilized a sweeping wire technique similar to the method described by Petrie and Pfender ${ }^{25}$ and which will be briefly discussed in this section. Kimura and Kanzawa $^{26}$ determined the total heat transfer to wires swept through a high intensity argon arc. In contrast to the studies of Ref. 24, the flow field in the arc induced by the cathode jet plays an important role as demonstrated by the results of Refs. 25 and 26. Kanzawa ${ }^{27}$ employed a water-cooled tube for determining the gross effect of electrical biasing on heat transfer. This method which uses the calorimetric principle for heat flux measurements is similar to that used by Meyer and Pfender ${ }^{28}$ although no attempt was made in Ref. 27 to evaluate local heat fluxes. Local heat flux measurements by using spherical thermocouple probes or cylindrical probes immersed into arc plasmas have been reported by Kanzawa. ${ }^{29,30}$ The results of these studies compare favorably with those which will be discussed in the following.

In order to experimentally assess the effect of ionization on heat transfer to a body immersed into or in contact with a plasma, either small diameter wires have been swept through a plasma generated by a free-burning arc or a thin water-cooled tube has been moved step by step through the plasma. Both methods will be briefly discussed without experimental details which have been presented elsewhere. ${ }^{25,28}$

\section{(A) Methods for measuring local heat fluxes}

The underlying principle for obtaining local heat fluxes is the same for both the sweeping wire technique and the water-cooled probe.

1. Sweeping wire technique. The wire probe consisting of $\mathrm{W}$ or $\mathrm{Pt}$ is swept with constant velocity through the plasma generated by a free-burning arc in argon atmosphere as shown schematically in Fig. 2. From the complete history of the resistance change of the wire, local heat fluxes to the wire averaged over its circumference and the temperatures of the wire can be derived. The rate of change of the resistance is related to the heat flow to the wire whereas the magnitude of the resistance reflects the temperature of the wire. With the known properties of the wire material, the heat flow to the wire $Q(x)$ is obtained where $x$ denotes the distance of the probe center from the plasma center. The measured distribution $Q(x)$ may be converted into local heat fluxes $q(r)$ using the Abel inversion technique similar to that utilized in spectroscopy. A prerequisite for the application of this method is rotational symmetry of the plasma. Since $T_{\text {plasma }} \gg T_{\text {wire }}$ the plasma temperature determines the heat transfer 


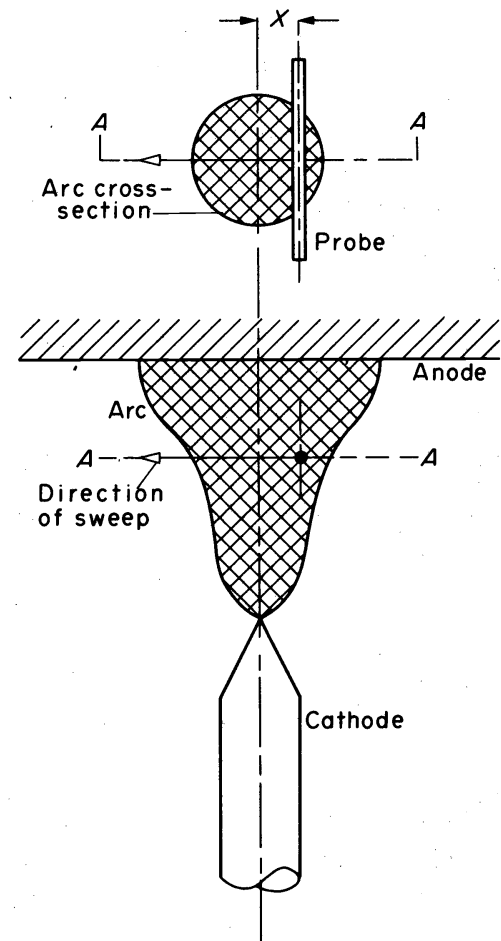

Fig. 2. Schematic of the sweeping wire technique applied to a free-burning arc.

independent of the wire temperature. The heat transfer from the plasma to the probe is adequately recorded if external and not internal conditions govern the heat transfer process. By specifying an external and internal resistance for heat transfer, one can show that $R_{\text {ext }} / R_{\text {int }}>$ 30 for the conditions in this experiment.

The effect of radiative heat transfer from the arc to the probe has been estimated. Taking $f f$-and $f b$-radiation into account as well as the shape factor of the wire with respect to the luminous arc, radiation contributes between $4-10 \%$ of the total heat transfer to the wire.

\section{Water-cooled probe}

As previously mentioned, the principle for obtaining local heat fluxes (Abel inversion) is the same as for the sweeping wire technique. The water-cooled probe, however, utilizes a calorimetric procedure for acquiring the raw data which yields higher accuracies of these data compared to the sweeping wire technique. In addition, biasing of the tube with various potentials is a straightforward procedure which allows the determination of the

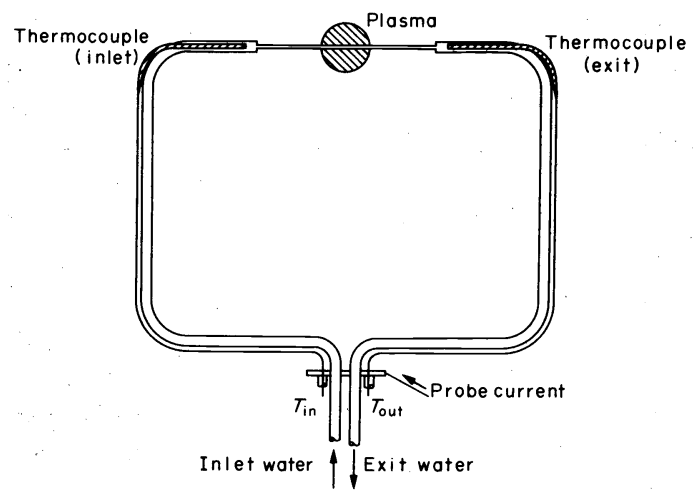

Fig. 3. Schematic of the water-cooled probe. effect of electric currents on heat transfer. Local values of heat fluxes and current densities can be measured simultaneously which facilitates comparisons with analytical correlations. In order to determine whether or not there is any effect associated with the catalyticity of the wall, the tube is coated with a thin layer $(20 \mu \mathrm{m})$ of pyrex glass.

Figure 3 shows a schematic of the probe in the mid-section of the arc plasma.

\section{(B) Experimental results}

For the evaluation of the data, the free stream properties of the arc plasma surrounding the probe are required. Plasma temperatures and velocities have been measured for 200 and $300 \mathrm{~A}$ arcs in argon at $p=1 \mathrm{~atm}$. The isotherm fields are shown in Fig. 4 and Fig. 5 shows the flow field induced by the cathode jet.

Close to the cathode the plasma temperature exceeds $2 \times 10^{4} \mathrm{~K}$ and the corresponding degree of ionization is almost $100 \%$. At a distance $5.5 \mathrm{~mm}$ from the cathode, the axis temperature in the arc drops to approximately $1.5 \times 10^{4} \mathrm{~K}$ and the associated degree of ionization is close to $50 \%$. The heat transfer measurements which will be discussed in the following have been taken over this range, i.e. from approximately $50-100 \%$ ionization.

In the case of the sweeping wire technique, bare metal wires ( $\mathrm{W}$ and $\mathrm{Pt}$ ) are swept with constant speed through the arc plasma in planes perpendicular to the arc axis at distances of approximately $1.5,3,4$ and $5.5 \mathrm{~mm}$ from the cathode tip. In this way, a wide range of plasma temperatures and velocities can be covered by the probe
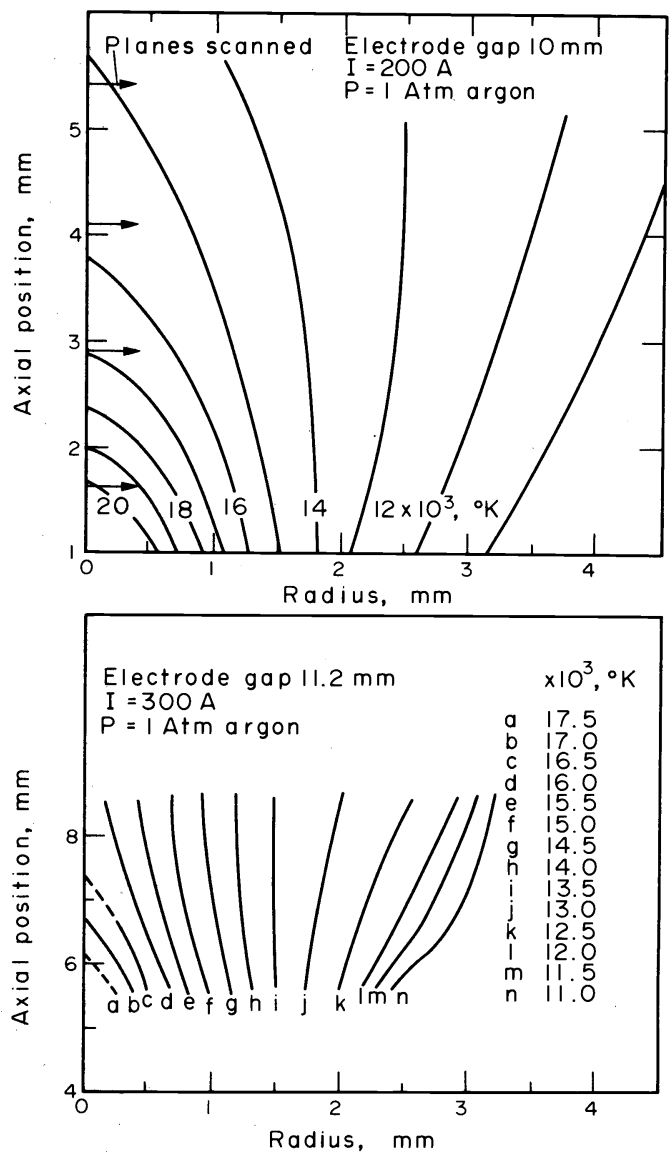

Fig. 4. Isotherm fields of 200 and $300 \mathrm{~A}$ free-burning arcs. 


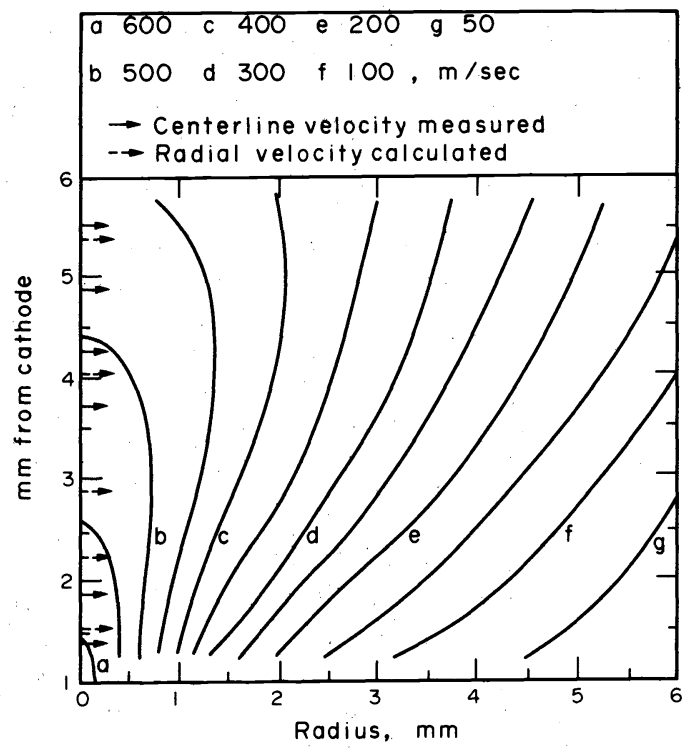

Fig. 5. Velocity field of the $200 \mathrm{~A}$ free-burning arc in argon atmosphere $(\mathrm{p}=1 \mathrm{~atm})$.

sweeps. Wire diameters of approximately $0.37,0.42,0.5$, 0.55 and $0.62 \mathrm{~mm}$ are utilized. For wire diameters $\leq 0.5 \mathrm{~mm}$, sweep velocities $\geq 25 \mathrm{~cm} / \mathrm{sec}$ are required to avoid melting of the center portion of the probes.
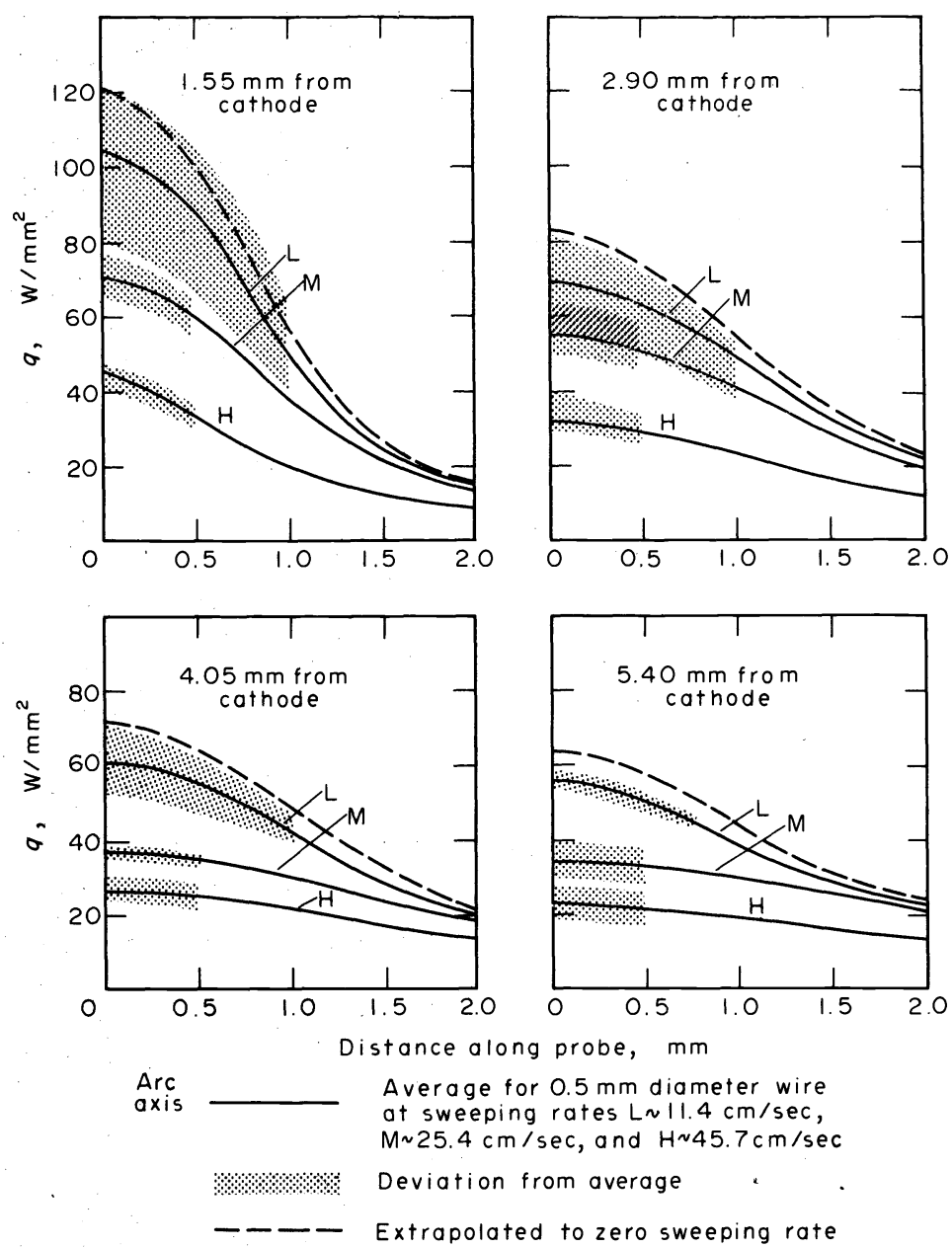

Fig. 6. Effect of probe diameter and sweeping rate on heat transfer to bare tungsten wires. are listed below.

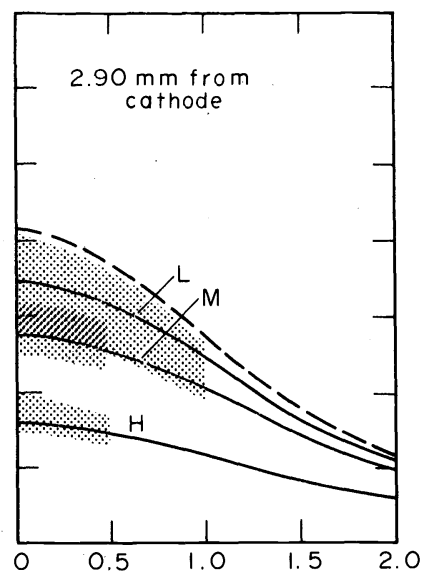

Considering convective heat transfer only, the Nusselt number is related to the Reynolds number by $\mathrm{Nu}_{d} \sim \operatorname{Re}_{d}{ }^{n}$. For Reynolds numbers in the range from 10 to 50 which are typical for these experiments, the exponent $n$ is approximately 0.5 so that $\mathrm{Nu}_{d} \sim d^{1 / 2}$ and $q \sim d^{-1 / 2}$ which agrees with the trend of the data. The effect of the sweeping rate has been eliminated by extrapolating the data to zero sweeping rate. A set of typical data is shown in Fig. 6 for distances from 1.55 to $5.4 \mathrm{~mm}$ from the cathode. The spread of the curves for various sweeping velocities indicates that the assumption of an equilibrium boundary layer is not consistent with this behavior. This finding is corroborated by the following consideration of characteristic time constants. The characteristic time for energy transport to the sweeping probe according to its velocity is $\tau_{\text {probe }}=10^{-3}-4 \times 10^{-3} \mathrm{sec}$. The corresponding time constants for an equilibrium and frozen boundary layer

\begin{tabular}{|c|c|}
\hline $\begin{array}{l}\text { State of boundary } \\
\text { layer }\end{array}$ & Characteristic time constant \\
\hline $\begin{array}{l}\text { Equilibrium } \\
\text { Frozen }\end{array}$ & $\begin{array}{l}\tau_{\text {equil }}=\Lambda_{\text {equil }}^{2} / \alpha=10^{-7}-10^{-5} \mathrm{sec} \\
\tau_{\text {frozen }}=\left(10 r_{p}\right)^{2} / D_{\text {amb }}=10^{-5}-10^{-3} \mathrm{sec}\end{array}$ \\
\hline
\end{tabular}

$\Lambda$ represents a characteristic diffusion length, $\alpha$ the thermal diffusivity, $r_{p}$ the probe radius and $D_{\text {amb }}$ the ambipolar diffusion coefficient. Since $\tau_{\text {frozen }}$ may reach the 
same order of magnitude as $\tau_{\text {probe}}$, the observed dependence of the heat flux to the probe on the sweeping rate is evidence that the boundary layer is not in an equilibrium state. According to this finding, the curves are extrapolated to zero sweeping rate as indicated by the dashed lines in Fig. 6.

The heat transfer, $Q(x)$, to the bare and pyrex coated water-cooled copper tube is determined for arc currents of 200 and $300 \mathrm{~A}$ and the measured distributions, $Q(x)$, are then inverted by the Abel inversion technique. In this way, circumferentially averaged local heat fluxes, $q(r)$, are obtained. Since any probe assumes an average floating potential in the plasma, the inversion of the heat flow does not result in the heat flux to a locally floating probe, but represents some effective heat flux, $q_{0}(r)$. Determination of heat fluxes to a truly locally floating surface requires a series of heat flow measurements with various biasing potentials.

For this purpose and for assessing the influence of a current flow to the probe on heat transfer, measurements of heat transfer and current-voltage characteristics are performed at a large number of $x$-positions using the water-cooled probe. An example of relevant results is shown in Fig. 7. From a set of such characteristics, a curve $I(x)$ for a given voltage is derived and inverted to local current densities, $j(r)$. Again, these current densities represent circumferentially averaged values. The described procedure is repeated for a family of voltages from which, finally, local current density-voltage characteristics can be derived for selected radii.

The effect of an electrical bias on the heat transfer expressed by $\Delta Q(x, V)$ which represents the difference between heat transfer at average floating potential to that at a given voltage, $V$, is tabulated and inverted as described before. The result is added to the effective heat flux, $q_{0}(r)$, resulting in the local heat flux, $q(r, V)$. The precision of fitting the data to a polynominal of least square type and then using the inversion necessitates a separation of the data into these two sets. Since the Abel inversion is a linear integral inversion of the function, a superposition as utilized here is permitted. The resolution of a direct inversion would not be sufficient to demonstrate the bias effect.

Local heat flux and effective heat flux for the pyrex and metal tube surfaces are shown in Fig. 8 for a plane $4.6 \mathrm{~mm}$

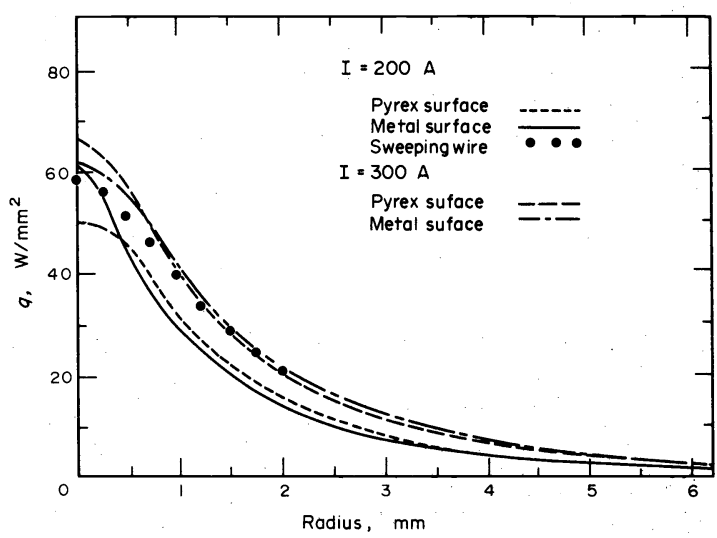

Fig. 8. Local and effective heat fluxes along various probes.

below the anode surface. For comparison, results obtained from the sweeping wire technique for the $200 \mathrm{~A}$ arc using bare tungsten wires are also included in this figure as dots. The agreement between the results obtained with these two different methods is within a few percent in the probe center but deviates appreciably for $r>0.5 \mathrm{~mm}$. Since the heat flux to an insulating surface (pyrex) is within experimental accuracy identical to that of a floating copper surface for the free-stream conditions of this experiment, it may be concluded that under these conditions all walls whether or not conducting behave as catalytic walls for recombination of ions with electrons. This conclusion is further corroborated by analytical considerations presented in Ref. 31 .

Figure 9 which refers to a biased tube shows the current density-voltage characteristics for four radii, and Fig. 10 shows the corresponding $\Delta q$ due to biasing as a function of the current density for three different radii. The probe characteristics of Fig. 9 reveal the typical shape of Langmuir characteristics. For $V \leq 8 \mathrm{~V}$, both sets of curves show ion saturation current. The heat flux (Fig. 10) changes in this region according to the change of voltage imposed on the ion saturation current. As the voltage approaches the floating point, the heat flux due to the saturated ion current rapidly diminishes merging into a flat region showing a minimum which is approximately $1 \%$ below the heat flux at floating potential and occurs one to

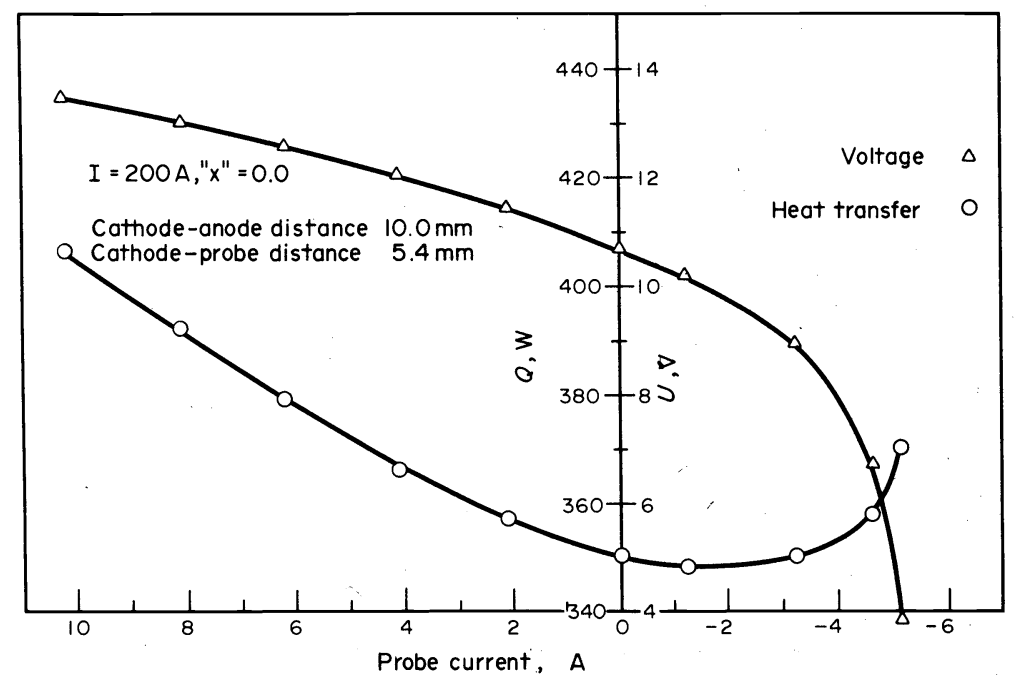

Fig. 7. Current-voltage and current-heat transfer characteristics of the water-cooled probe. 

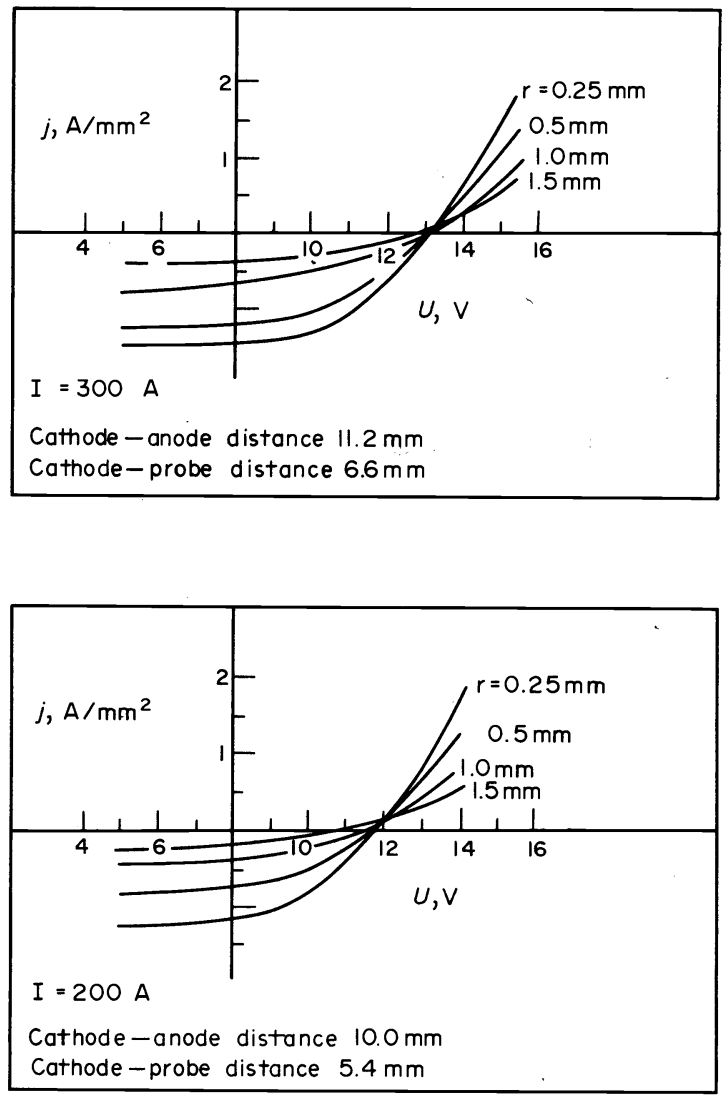

Fig. 9. Abel-inverted current-voltage characteristics obtained with the water-cooled probe. two volts below the floating potential. Beyond this minimum, the heat flux to the probe increases with increasing voltage caused by the increasing electron flux to the probe. At the point of floating potential, the curve $\Delta q(j)$ reaches a slope of approximately $4-5 \mathrm{~V}$ corresponding to the work function of the probe material (copper). The slope continues to increase with increasing voltage. These trends compare favorably with analytical results which will be discussed in the next paragraph.

The curves of Fig. 10 reveal also that $\Delta q$ for local floating conditions is very small compared to $q_{0}$, the effective heat flux (for a metallic probe on average floating potential). This finding has significant consequences for experimental heat transfer studies in plasmas. The desired local value of heat transfer can be found with a high degree of accuracy by simply inverting the average floating heat transfer distribution, $Q(x)$.

\section{(C) Comparison of experimental results with theoretical predictions}

The first part of the following analysis deals with the verification of the frozen chemistry indicated by experimental results. In the second part, a possibility for establishing a semiempirical relationship for heat transfer from a plasma to a solid body will be discussed; and in the third part, analytical predictions for heat transfer to biased walls will be compared with the corresponding experiments using the water-cooled probe.

1. Thermodynamic state of the boundary layer surrounding a probe. Based on thermal conductivities and diffusion coefficients presented by Fay and Kemp, ${ }^{32}$ the temperature distribution and the electron density in the region in front of the probe which is governed by conduction and diffusion can be calculated for the two
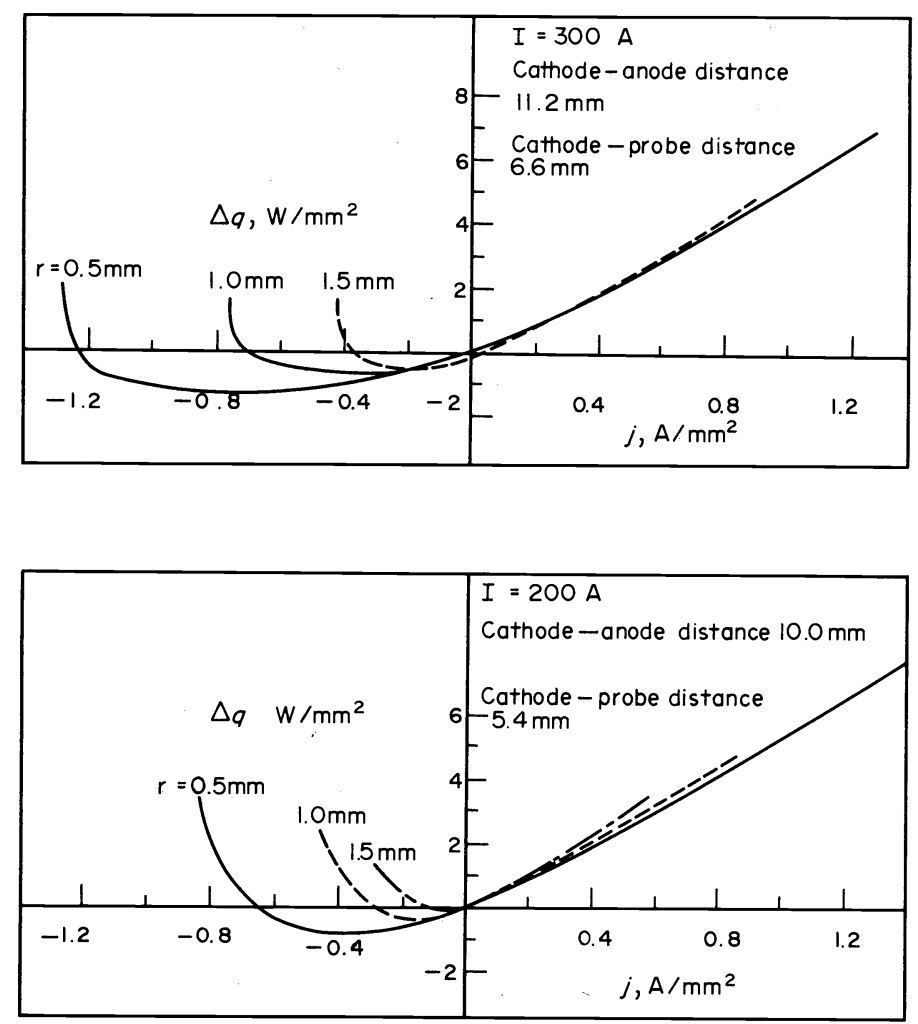

Fig. 10. Heat flux-current density characteristics obtained with the water-cooled probe. 
extreme cases of chemical equilibrium and frozen chemistry. For this analysis, the following assumptions have been introduced:

(a) Steady-state, one-dimensional heat flow.

(b) Kinetic equilibrium exists among the various species (electrons, ions, neutrals).

(c) Radiation heat transfer from the plasma to the probe surface is neglected.

(d) The wall is assumed to be fully catalytic.

(e) The calculation is performed for a given wall heat flux.

(f) The sheath is excluded from this analysis.

Results for a wall heat flux of $3 \mathrm{~kW} / \mathrm{cm}^{2}$ are presented in Fig. 11. As expected, the electron density distribution changes significantly for frozen conditions in the boundary layer which has a thickness in the order of $10^{-2} \mathrm{~cm}$. A conservative estimate of the diffusion transit time through the boundary layer and of the recombination time demonstrates that they are of the same order of magnitude which clearly indicates that deviations from chemical equilibrium have to be expected. By defining a ratio

$$
\gamma=n_{i} v_{i}\left(\frac{5}{2} k T_{\text {ref }}+\mathrm{e} V_{i}\right) q_{y}
$$

of the effective heat transfer due to diffusion of ion pairs to and recombination at the surface to the heat transfer arising from diffusion and conduction, the relative importance of the charged particles for this heat transfer process can be assessed, assuming that the boundary layer is completely frozen. In eqn (20) $n_{i}$ represents the ion density, $v_{i}$ the diffusion velocity, $T_{\text {ref }}$ the free-stream temperature, and $V_{i}$ the ionization potential. For a number of free-stream temperatures within the range of experimental values, $\gamma$ has been calculated and the result is shown in Fig. 12. From known free-stream temperatures (Fig. 4), $T_{\text {ref, }}$ and measured local heat fluxes, $q_{y}$, the product $n_{i} v_{i}$ or the ion current density may be found from Fig. 12 and compared with measured ion saturation current densities. This comparison is shown in Table 1. The experimental values are consistently higher than the calculated ones which is not surprising because the calculation refers to a wall at floating potential at which the ion current is already $20-30 \%$ reduced compared with its actual saturation value. Taking this fact into account

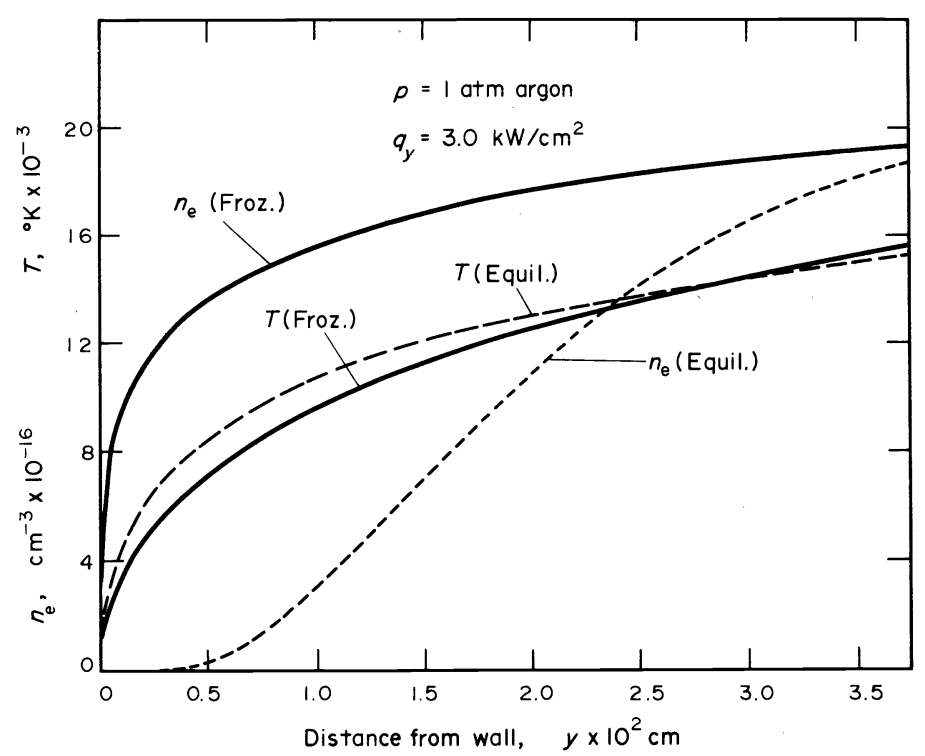

Fig. 11. Calculated electron density and temperature distributions in a plasma-wall boundary layer.

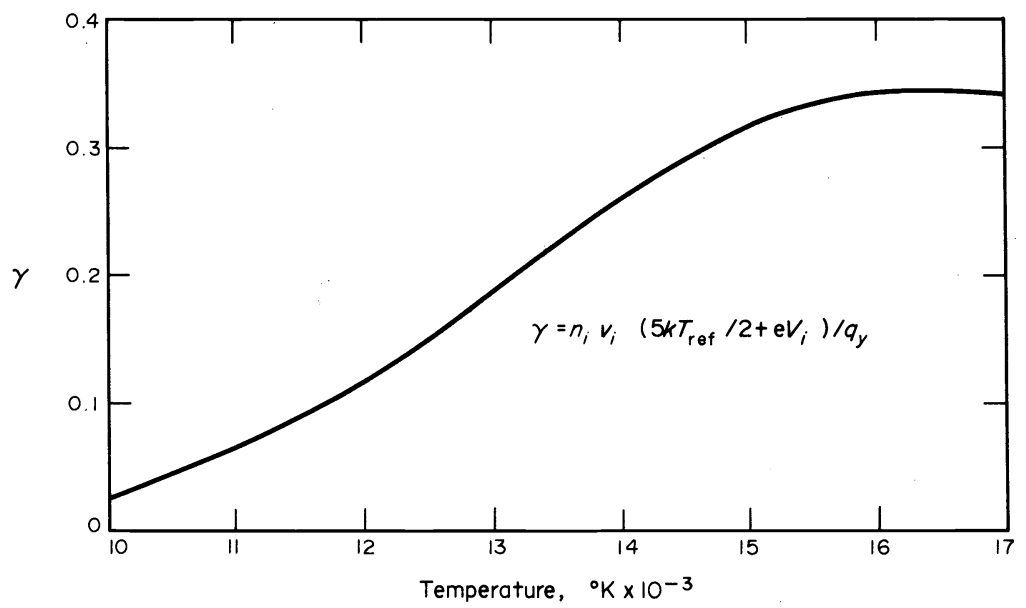

Fig. 12. Ratio of heat transfer by diffusion to total heat transfer. 
Table 1. Comparison of experimental and calculated ion saturation currents

\begin{tabular}{ccccccc}
\hline & $\begin{array}{c}r \\
(\mathrm{~mm})\end{array}$ & $\begin{array}{c}T \\
\left({ }^{\circ} \mathrm{K}\right)\end{array}$ & $\begin{array}{c}q_{y} \\
\left(\mathrm{~W} / \mathrm{mm}^{2}\right)\end{array}$ & $\begin{array}{c}j_{i, \text { sat }} \text { Calc } \\
\left(\mathrm{A} / \mathrm{mm}^{2}\right)\end{array}$ & $\begin{array}{c}j_{i, \text { sat }} \text { Exp } \\
\left(\mathrm{A} / \mathrm{mm}^{2}\right)\end{array}$ & $\begin{array}{c}\text { Deviation } \\
\%\end{array}$ \\
\hline$I=200 \mathrm{~A}$ & 0.5 & 14800 & 45 & 0.73 & 0.87 & 16 \\
& 1.0 & 13500 & 30 & 0.36 & 0.48 & 25 \\
& 1.5 & 12300 & 21 & 0.16 & 0.23 & 30 \\
\hline$I=300 \mathrm{~A}$ & 0.5 & 15400 & 56 & 0.97 & 1.27 & 24 \\
& 1.0 & 14100 & 41 & 0.58 & 0.78 & 26 \\
& 1.5 & 13000 & 29 & 0.30 & 0.47 & 36 \\
\hline
\end{tabular}

the agreement between experiment and analysis suggests that the boundary layer is frozen.

Further evidence for frozen chemistry is demonstrated by the results of the sweeping wire technique. An upper and a lower limit for the heat transfer to the wire is obtained by assuming either a completely frozen or an equilibrium state. Figure 13 shows the corresponding comparison which, again, indicates that frozen conditions are closely approached.

2. Semi-empirical dimensionless heat transfer correlations. From a dimensionless analysis ${ }^{33}$ follows that the heat transfer due to conduction and diffusion can be expressed by a dimensionless heat transfer coefficient

$$
\mathrm{Nu}_{d, f}=f\left(\operatorname{Pr}_{f}, \operatorname{Re}_{d}, S c, \xi, \phi\right) .
$$

In this equation, the index $f$ denotes frozen condition, $S c$ represents the Schmidt number, $\xi$ the degree of ionization, and $\phi$ a dimensionless expression $\left(\phi=\mathrm{e} V_{F} / k T\right)$ for the floating potential $V_{F}$.
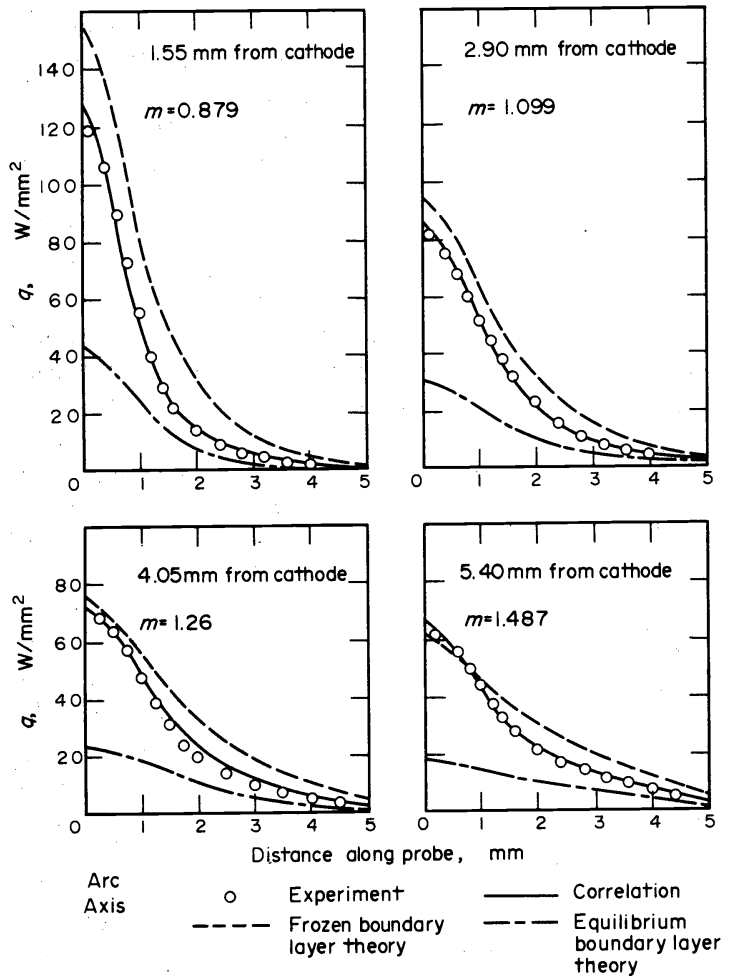

Fig. 13. Comparison of sweeping wire data with chemical equilibrium and frozen chemistry limits (in Ref. to eqn 22).
An explicit correlation derived for this situation is ${ }^{33}$

$$
\mathrm{Nu}_{d, f}=\left[0.064 \operatorname{Pr}_{f}^{1 / 3}+0.0348\left(\xi \phi^{2} / S c\right)^{m}\right] \operatorname{Re}_{d}^{0.769}
$$

where the exponent $m$ still depends on the particular conditions prevailing in a given sweeping plane. The solid lines in Fig. 13 are based on eqn (22) and represent the data within $\pm 15 \%$ over the entire range of experimental conditions.

3. Heat transfer model for heat transfer to a biased wall. A simplified heat transfer model should allow to explain the observed heat flux dependence on current. For this model, the following assumptions are introduced:

(a) Steady state prevails;

(b) The voltage drop in front of the wall is essentially confined in the sheath which is assumed to be collisionless;

(c) The electrons follow a Maxwell-Boltzmann distribution;

(d) The model holds only for wall potentials less or equal to the floating potential;

(e) The accommodation coefficient for the ions is assumed to be one.

For a differential change of the current density, the corresponding change of the wall heat flux may be expressed by

$$
\mathrm{d} q=-j_{i, s a t} \mathrm{~d} U_{w}+\mathrm{dj} \mathrm{j}_{\mathrm{e}}\left(\phi_{w}+2 k T_{e s} / \mathrm{e}\right) .
$$

In this equation $j_{i \text { sat }}$ represents the ion saturation current density, $U_{w}$ the wall potential, $j_{e}$ the electron density, $\phi_{w}$ the work function of the wall material, and $T_{e s}$ the electron temperature at the sheath edge. Equation (23) may be written as differential equation

$$
\frac{\mathrm{d} q}{\mathrm{~d} U_{w}}=-j_{i, \text { sat }}+\left(\frac{\mathrm{d} j_{e}}{\mathrm{~d} U_{w}}\right)\left(\phi_{w}+2 k T_{e s} / e\right) .
$$

At probe potentials sufficiently negative with respect to the surrounding plasma, the ion current drawn by the probe becomes saturated and at this point $\mathrm{dj}_{e} / \mathrm{d} U_{w}$ is negligible, i.e. only the first term in eqn (24) has to be considered in agreement with experimental data. With assumption (c), eqn (23) can be written in differential form as

$$
\frac{\mathrm{d} q}{\mathrm{~d} j_{e}}=-\left(j_{\mathrm{i}, \mathrm{sat}} / j_{e}\right)\left(k T_{e s} / e\right)+\phi_{w}+\overline{2} k T_{e s} / e .
$$

This equation simplifies to

$$
\frac{\mathrm{d} q}{\mathrm{~d} j_{e}}=\phi_{w}+k T_{e s} / e
$$

for the point of floating potential. At the point of minimum 
heat transfer (i.e. $\mathrm{d} q / \mathrm{d} j_{e}=0$ ), one finds

$$
j_{i, s a t} / j_{e}=e \phi_{w} / k T_{e s}+2
$$

or

$$
\Delta U_{w}=\left(k T_{e s} / e\right) \ln \left[\left(e \phi_{w} / k T_{e s}\right)+2\right]
$$

where $\Delta U_{w}$ represents the difference between floating potential and the point of minimum heat transfer.

The derived relations may be used for the interpretation of experimental data. Although the experimental data do not precisely agree with the model, reasonable agreement exists over the entire range of currents. Assuming bounding values for $T_{e s}$ between 5000 and $15,000^{\circ} \mathrm{K}$, a comparison between experimental results and predictions based on the adopted model are feasible.

Equation (26) implies that the slope at floating potential should be approximately $4-5 \mathrm{~V}$ in good agreement with the slopes of Fig. 10. Equation (28) requires that $\Delta U_{w}$ should be approximately $1-2 \mathrm{~V}$ in reasonable agreement with the differences obtained from Figs. 9 and 10.

\section{ELECTRODE HEAT TRANSFER}

\section{(A) General considerations}

The energy transfer process becomes more involved to a surface which draws substantial electric currents from the adjacent plasma (electrodes). This situation prevails, for example, at the electrodes of arc gas heaters or arc furnaces which are attracting increasing interest. A high intensity $\operatorname{arc}(I>100 \mathrm{~A})$ at atmospheric or higher pressures usually serves as the heat source in these devices.

Heat transfer to the electrodes is strongly influenced by the properties and the behavior of the plasma adjacent to the electrodes, the so-called electrode regions.

In spite of intensive research over the past decades the electrode regions are still poorly understood. This lack of understanding is a consequence of the complexities prevailing in these regions caused by the interaction of electrical, magnetic, thermal, and fluiddynamic effects, in addition to surface effects which are difficult to assess and frequently impossible to control. Considering these facts, it is not surprising that there is still no comprehensive theory capable of describing all the observed phenomena in these regions.

Gathering of experimental data from the electrode regions faces similar problems. The extreme values of temperature, current density, field strength etc. prevailing in these regions and the steep gradients of these parameters make diagnostics a formidable task. Furthermore, these extreme conditions in the electrode regions may cause deviations from LTE which creates another problem for the interpretation of experimental data. It is even doubtful whether or not the velocity distribution of the various plasma components (electrons, ions, neutrals) is still Maxwellian close to the electrodes.

In the case of electrode spots (extremely constricted current attachments), the highly disturbed surface state contributes further to this complexity. One may consider the location of an electrode spot as a highly disturbed metal lattice or as an extremely dense metal plasma. The electrode surface at the location of such a spot does not exist any more; it becomes a transition zone between solid state and probably a non-ideal gaseous plasma. These facts explain why there are only very little and rather scattered experimental data available in the literature about the electrode regions. Many findings are more or less concerned with phenomenological observations only.

Nevertheless, the literature dealing with the electrode regions of electric arcs is very extensive, in particular the cathode region attracted great interest because of the strong impact of the effects in the cathode region on the entire discharge. Pertinent literature references on the electrode regions up to 1955 are given by Finkelnburg and Maecker. ${ }^{34}$ A more comprehensive review listing 645 references has been published by Ecker ${ }^{35}$ summarizing relevant work on this subject up to 1959. Ecker stresses in his treatment the basic physics involved in the electrode regions with emphasis on low intensity arcs, whereas Guile $^{36}$ in a later review which includes 238 references emphasizes those aspects which are of interest for engineers involved in arc applications, particularly in the application of high intensity arcs. In a recent review by Kimblin $^{37}$ experimental aspects of the anode region are discussed for vacuum and atmospheric pressure arcs with emphasis on the effect of plasma flow from the cathode.

Since the total heat fluxes to the anode are usually larger than to the cathode, the following discussion will be mainly concerned with anode heat transfer in high intensity (thermal) arcs.

The anode region, as any other part of an electrical discharge, is governed by the conservation equations including the current equation. Unfortunately, any attempt to solve these equations for the anode region faces three major problems. First of all, the conventional conservation equations apply only as long as the continuum approach is valid. Since the anode fall spacing is in the order of one mean free path length of the electrons the continuum approach is no longer valid for that part of the anode region. Secondly, the application of the conservation equations requires that the plasma is in LTE or at least that its thermodynamic state is known. There is serious doubt that LTE exists for the entire anode region. Close to and in the anode fall region deviations from LTE are anticipated due to differences in electron and heavy particle temperatures and due to deviation from chemical composition equilibrium. Finally, the specification of realistic boundary conditions for an analysis of the anode region is extremely difficult.

The principle task of the anode fall, namely to provide an electrical connection between the high temperature plasma of the arc and the low temperature anode embraces several effects imposed by the conservation equations. Conservation of energy requires, for example, that field strength and current density adjust themselves so that the total net energy losses suffered by a volume element are compensated for by the ohmic heating in the same volume element. Because of the steep gradients of the plasma parameters in front of and normal to the anode surface, losses in axial direction may be substantially larger than in radial direction.

Conservation of charge carriers demands ion production in the anode fall which, however, amounts to only a small fraction (approx 1\%) of the total current. At the anode surface the current is exclusively carried by electrons (disregarding ion emitting anodes) which gives rise to a net negative space charge. This space charge tapers off with increasing distance in normal direction from the anode due to ion production mentioned before, until the unperturbed state in the plasma column is reached. The potential drop in the anode fall zone is a consequence of the net space charge adjacent to the anode surface (Poisson equation).

As shown in Ref. 1, heat transfer by the electrons 
entering the anode frequently represents the dominant heat transfer mechanism at the anode. Therefore, the current density distribution at the anode and the specific anode heat flux distribution are closely related.

The attachment of the arc at the anode surface may occur diffusely as well as severely constricted (spot) and it is still not entirely clear under which conditions constriction will occur. Chemical reactions on the anode surface as, for example, encountered in arcs operated in atmospheric air or in other oxidizing fluids seem to favor a constricted anode root which, at the same time, may travel more or less randomly over the anode surface with appreciable velocities. Anode evaporation is another mechanism which leads, in general, to spot formation. In high current arcs with relatively small electrode separation (a few centimeters) the diffuse anode attachment is directly associated with the cathode jet. The well known bell shape of a free-burning high intensity arc is a typical example. The intense cathode jet impinging on the anode surface pushes hot plasma against the anode eliminating the need for ionization in the anode fall zone. By increasing the electrode gap under otherwise identical conditions the influence of the cathode jet at the anode is diminished and, finally, at sufficiently large gaps the arc forms a single or several spots at the anode surface.

These findings suggest that the anode attachment is governed by the thermal conditions at and adjacent to the anode surface. Any effect which has a favorable influence on the energy balance in the anode region, in the sense that internal heat generation by the arc may be decreased, seems to favor a diffuse arc attachment. In a suitable arc configuration the cathode jet is able to provide a continuous flow of hot plasma into the anode region reducing in this way the necessity of heat generation by the arc itself. The correlation between current densities and heat fluxes follows from an anode energy balance. Local heat fluxes to the anode may be expressed by ${ }^{1}$

$$
q_{a}=q_{c}+q_{r}+j_{e}\left(\frac{5}{2} \frac{k T e}{e}+V_{a}+\phi_{a}\right)
$$

In this equation $q_{c}$ stands for local heat fluxes by conduction and convection, $q_{r}$ represents the radiative heat flux from the plasma, and the last term describes the energy transferred by the electrons to the anode. $T_{e}$ is the temperature of the electrons entering the anode fall zone, $V_{a}$ is the anode fall, $\phi_{a}$ the work function of the anode material, and $j_{e}$ the electron current density at the anode surface. In a steady-state situation the energy gain according to eqn (29) must be balanced by losses which may consist of conduction and convection to the surrounding gas, radiation from a single or several hot anode spots, and energy losses due to ablation and vaporization of anode material.

In most cases of practical importance heat transfer to the anode is governed by the electron flow (last term in eqn (29) i.e.

$$
q_{a} \simeq \mathrm{j}_{e}\left(\frac{5}{2} \frac{k T e}{e}+V_{a}+\phi_{a}\right)
$$

and

$$
\frac{q_{a}}{j_{e}}=U_{I} \simeq\left(\frac{5}{2} \frac{k T e}{e}+V_{a}+\phi_{a}\right) .
$$

For known values of $U_{I}$ the anode heat flux follows from measured current densities.
Since the arc attachment at the anode may be diffuse or sharply constricted, the corresponding current densities range from $10^{2}$ to $10^{5} \mathrm{~A} / \mathrm{cm}^{2} .^{38-41}$ These results appear to be consistent with anode heat flux measurements ${ }^{42-45}$ which will be discussed in the following paragraph.

\section{(B) Anode heat flux measurements}

In this paragraph recent results of anode heat transfer studies conducted in this laboratory will be reviewed. Results of earlier studies are summarized in Ref. 1 .

1. Anode heat transfer in an arc with superimposed axial flow. In these experiments an arc configuration consisting of two plane anodes has been used as shown schematically in Fig. 14. This arrangement resembles the electrode configuration of actual arc gas heaters or plasma torches, and allows unobstructed viewing of the entire arc. The surface area of the anodes is very large compared to the size of the arc attachment at the anode. The location, motion, and cross-sectional area of the anode arc attachment are determined by a balance of-gas dynamic, electric, and magnetic forces as well as thermal effects. This special anode geometry enables the measurement of the size of the near-anode arc column and the associated average heat flux densities due to the electron current entering the anode.

Under the influence of the superimposed flow the arc attachment at the anode may remain stationary or move downstream increasing the arc length until arc shunting occurs close the cathode. ${ }^{46,47}$ Since this process repeats itself periodically, the arc operates in this case in a "fluctuating mode".

The motion of the anode root leads to changes of the cross-sectional area and shape of the near-anode arc column. These changes are a consequence of the interaction of gas flow and self-magnetic field within the boundary layer overlying the anode surface. The velocity of travel and the relative change of the size of the near-anode arc column as a function of various arc parameters have been measured with an optical method and a floating potential probe. ${ }^{44}$

The heat flux measurements for a current range from 50 to $200 \mathrm{~A}$, in argon at pressures from approx 0.1 to $1 \mathrm{~atm}$ and flow velocities from 0 to $100 \mathrm{~m} / \mathrm{sec}$ demonstrate that $70-90 \%$ of the heat flux to the anode is transmitted at the anode root, in spite of the relatively large anode surface area exposed to the arc plasma. This concentration of the heat flux at the anode root gives rise to very high specific heat fluxes which can be found from measurements of the size of the anode root.

With the previously mentioned diagnostic techniques the size of the near-anode column, which may be in a first approximation equaled with the size of the anode root, has been measured parallel and perpendicular to the flow direction. The results show that the anode root is elongated in flow direction and the elongation increases with increasing flow velocities for pressure $p \geq 0.4 \mathrm{~atm}$. Average current densities in the attachment spot are in the

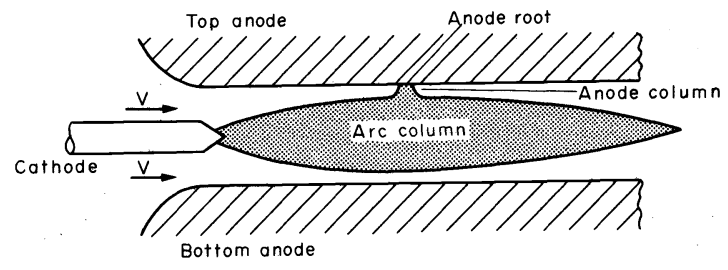

Fig. 14. Schematic of an open arc configuration. 
range from 120 to $520 \mathrm{~A} / \mathrm{cm}^{2}$ and the corresponding average specific heat fluxes range from approx $1-6 \mathrm{~kW} / \mathrm{cm}^{2}$.

In general, the pressure exerts a strong influence on the specific heat fluxes, which show a rapid increase with increasing pressure. The maximum specific heat flux at the anode root may well be an order of magnitude higher than the average value. Measurements of maximum specific heat fluxes at the anode of high intensity arcs will be discussed in the following paragraph.

2. Measurement of maximum specific heat fluxes at the anode of high intensity arcs. Anode heat fluxes play an important role in the design of all types of arc devices, they must either be minimized for achieving high efficiencies for example in arc gas heaters and plasma torches, or they must be maximized and shaped for applications such as welding, cutting or melting of materials. The maximum specific heat fluxes are of particular importance because they frequently determine integrity or failure of an electrode. For this reason a method has been developed in this laboratory which permits reliable measurement of maximum specific heat fluxes at the anode of pulsed arcs in the $\mathrm{kA}$ range. ${ }^{45}$

The measurement is accomplished by recording the light emission from the rear face of a very thin anode with a calibrated light fiber-photomultiplier combination. The calibration of this arrangement provides a correlation between photomultiplier output and rear surface temperature of the anode. Advantages of this system include the fact that it provides a completely passive temperature measurement with response times at least an order of magnitude faster than needed. Furthermore, use of the light fiber eliminates problems with electrical noise and high voltages. This arrangement provides a temperature history of the anode rear face. The anode rear face temperature may be calculated for assumed input heat fluxes and the calculated temperature-time responses may be compared with the experimental results. In this way the anode heat fluxes are deduced from the best fit with the experimental temperature histories.

The results obtained with this technique apply for steady state as well as for transient arcs because the arc reaches a quasi-steady state approx $10 \mu \mathrm{sec}$ after its initiation. The peak (central) heat fluxes are measured as the light fiber is experimentally determined to be in a central isothermal region of the anode. Spatial variations

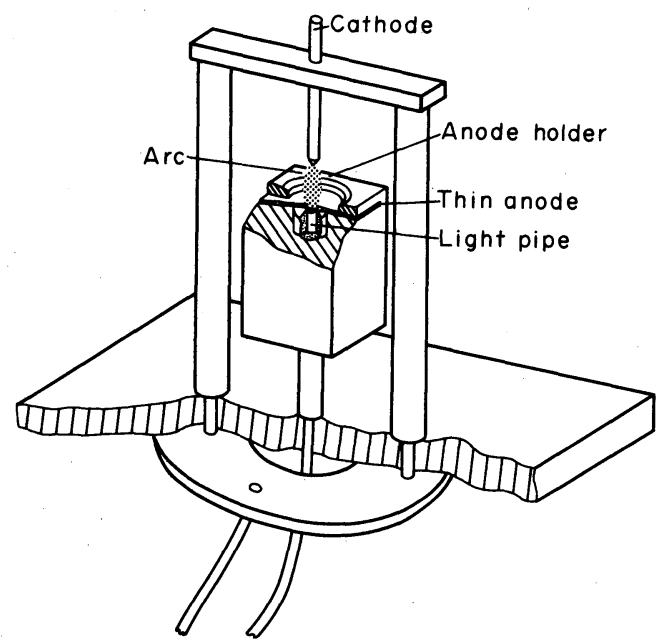

Fig. 15. Schematic of the electrode and light fiber arrangement. on the anode could be studied with multiple probes as an extension of the method described in this paper.

Figure 15 shows a sketch of the electrode arrangement including the light fiber optics. It is essential that the current is fed to the electrodes in a symmetric manner to avoid self-induced arc motion. The anode consists of a thin metal foil $(0.025-0.075 \mathrm{~mm}$ thick) arranged perpendicularly to axis of the cathode. The light fiber optics aligned with the axis of the cathode views the rear face of the anode. The high heat fluxes from the arc cause the surface temperature to rise very rapidly with little penetration into the material. Therefore, only short duration $\operatorname{arcs}(\leq 200 \mu \mathrm{sec})$ can be used if surface integrity is to be maintained.

The anode temperature history is obtained from the photo-multiplier output by a calibration procedure. The light fiber is exposed to an electrically heated metal strip which is identical to the anode surface under identical geometrical conditions. The temperature of the surface is monitored with thermocouples and the photomultiplier output is recorded. Figure 16 shows a typical calibration curve for a Cu-anode.

Each firing of the arc is photographed with a high speed streak camera (time resolution approximately $10^{-7} \mathrm{sec}$ ). These photographs provide a time history of a crosssection of the arc very close to the anode. They serve two functions: first they give an indication of the initial transient period of the arc, and second they provide an independent observation of the arc in the immediate vicinity of the anode.

As previously mentioned the heat flux is determined by matching experimental and analytical anode temperature histories. The top anode surface begins to rise in temperature immediately upon arc initiation. After a delay, associated with the thermal diffusivity of the anode material and with the anode thickness, the rear surface temperature begins to rise in temperature also. It is this temperature history which is experimentally measured and which must be analytically determined.

The analytical solution incorporates temperature dependent properties of the anode material and allows for possible surface melting. The approach for avoiding the discontinuity at the liquid-solid interface assumes that materials melt over a small but finite temperature range rather than at a particular temperature. This assumption reduces the melting problem to a conduction problem of a single phase with temperature dependent properties. In particular, the latent heat of fusion appears as an increased specific heat near the melting temperature.

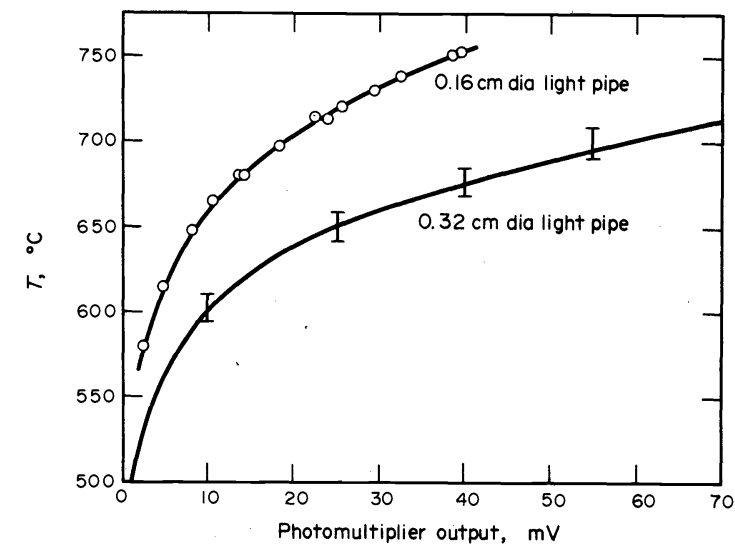

Fig. 16. Calibration of optical arrangement for $\mathrm{Cu}$-anode. 


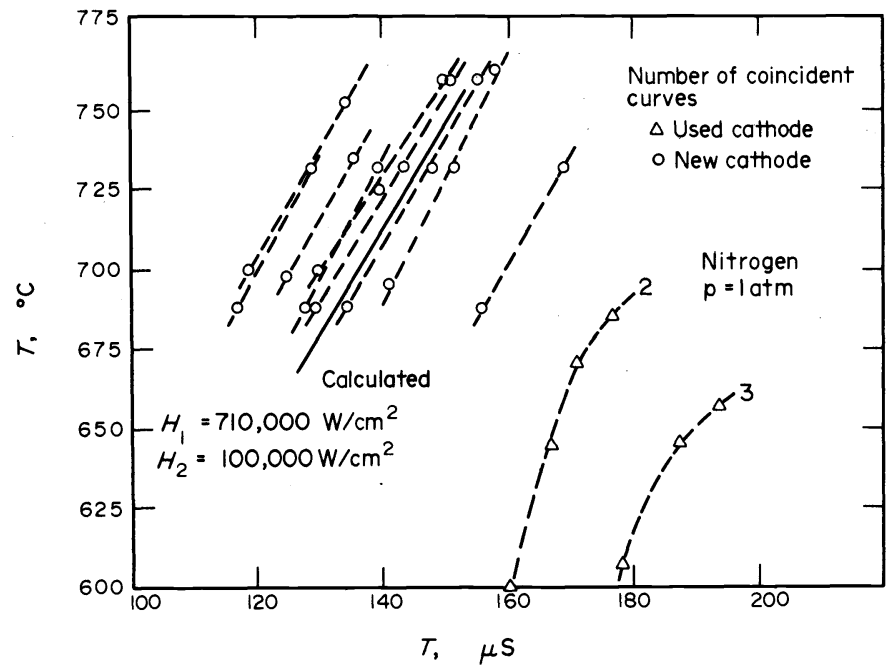

Fig. 17. Data for $5 \mathrm{kA}$ arc with $0.076 \mathrm{~mm} \mathrm{Cu}$-anode.

There is a finite time before the photomultiplier begins to respond. This is due to both the thermal diffusion time through the anode, and the fact that the photomultiplier typically begins to respond at $T \geq 500^{\circ} \mathrm{C}$. The result is that the heat flux during the early part of the arc can be determined only as a time integrated quantity. High speed streak photographs indicate a transient period of approximately $10 \mu \mathrm{sec}$ followed by a steady arc. In order to establish a correlation with experimental conditions, a two heat flux analytical model is adopted. An initial influx, $H_{1}$, is assumed to prevail for the first $10 \mu \mathrm{sec}$ followed by a heat flux $\mathrm{H}_{2}$ thereafter. This two flux step model is found to result in temperature histories which agree very well with experimentally measured histories.

As an example Fig. 17 shows a number of measured temperature-time histories (dashed lines) and a calculated curve (solid line). The data generally indicate that the transient heat flux during the first $10 \mu \mathrm{sec}$ exhibits considerable scatter whereas the steady heat flux is quite reproducible. The high heat flux during the initial phase of the arc is mainly due to the severe constriction of the arc column revealed by streak photographs. As the arc diameter increases a quasi-steady state is reached with substantially lower local heat fluxes. The initial phase of the arc is rather erratic and this fact is reflected in the data scatter. The critical parameter, however, is the slope of the curves. This slope provides the magnitude of the local heat flux at the time when the temperature is being measured $\left(\mathrm{H}_{2}\right)$. The position of the curves relates to the initial heat flux $\left(H_{1}\right)$. Because of the statistical nature of the breakdown a certain scatter of the data has to be expected.

Tungsten, molybdenum, titanium and copper anodes have been utilized with argon and nitrogen at atmospheric pressure as working fluids (the combination titaniumnitrogen has been excluded because of chemical reactions) and arc currents of $5 \mathrm{kA}$. The initial transient heat fluxes in the range from 170 to $700 \mathrm{~kW} / \mathrm{cm}^{2}$ are followed by quasi-steady heat fluxes ranging from 25 to $100 \mathrm{~kW} / \mathrm{cm}^{2}$. The heat fluxes are functions of both the cathode condition and the working gas, but not of the anode material as long as the anode surface conditions are not disturbed. For argon as working fluid the maximum specific heat fluxes are by a factor of 2-4 lower than the corresponding heat fluxes in nitrogen.
Acknowledgements-Most of the work summarized in this paper has been supported by the National Science Foundation under the following grants: NSF-GK 1728, NSF-GK 15924, and NSF-GK 35230 .

\section{REFERENCES}

${ }^{1}$ E. R. G. Eckert and E. Pfender, Advances in plasma heat transfer, in Advances in Heat Transfer. Vol. 4, Academic Press, New York (1967).

${ }^{2} \mathrm{C}$. Bonet, M. Daguenet and P. Dumargue, Etude theorique de l'evaporation d'une particule spherique d'un materiau refractaire dans un plasma thermique, Int. J. Heat Mass Transfer 17, 643 (1974).

${ }^{3} \mathrm{C}$. Bonet, M. Daguenet and P. Dumargue, Etude theorique de l'evaporation d'une particule spherique d'un materiau refractaire dans un plasma thermique, Int. J. Heat Mass Transfer 17, 1559 (1974).

${ }^{4} U$. Landt, Entwicklungen auf dem Gebiet der anorganischen Plasmachemie Teil 1: Reaktionen im Plasmastrahl, Chemie-Ing.Tech. 42 (9/10), 617 (1970).

${ }^{5}$ R. Neubeck and F. Fetting, Einfluss von Vermischung und Temperatur auf chemische Reaktionen im Plasmastrahl, Chemie-Ing.-Tech. 42 (7) 439 (1970).

${ }^{6}$ I. G. Sayce, Plasma processes in extractive metallurgy, Adv. Ext. Met. Refining, Proc. Int. Symp. 241 (1971).

${ }^{7} \mathrm{M}$. L. Thorpe, High-temperature technology and its relationship to mineral exploitation, Adv. Ext. Met. Refining, Proc. Int. Symp. 275 (1971).

${ }^{8}$ S. Katta and W. H. Gauvin, The effect of local gas velocity and temperature on local heat transfer to a sphere in a high temperature jet, Can. J. Chem. Eng. 51 (3) 307 (1973).

${ }^{9}$ I. Preece and C. W. D. Andrews, Plasma spraying of ferrites, $J$. Mat. Sci. 8, 964 (1973).

${ }^{10} \mathrm{~F}$. H. Howie and I. G. Sayce, Plasma heating of refractory melts, Rev. Int. Htes Temp. Refract. 11, 169 (1974).

The following references (11-21) refer to papers presented at the 168th National ACS Meeting, Atlantic City, N.J., 8-13 Sept., 1975.

${ }^{11}$ M. G. Fey and F. J. Harvey, The role of plasma heating devices in the electric economy.

${ }^{12}$ D. R. MacRae, Criteria for plasma processing in extractive metallurgy.

${ }^{13}$ J. D. Chase, J. F. Skrivan, D. Hyman and J. E. Longfield, Plasma jet process for making elemental phosphorous.

${ }^{14}$ H. Krause, Jr., E. J. Mezey and W. M. Goldberger, A comparison of DC-Arc, $\mathrm{RF}$ and microwave plasma generators for production of $\mathrm{P}_{4} \mathrm{O}_{4}$ and phosphine.

${ }^{15}$ M. F. Barrett, F. H. Howie and I. G. Sayce, Plasma fuming of tin slags.

${ }^{16} \mathrm{~J}$. Lawton, Titanium winning by a plasma process. 
${ }^{17} \mathrm{~W}$. H. Gauvin, Fundamental and engineering aspects of reactor design for plasma processes.

${ }^{18} \mathrm{M}$. Thorpe, Zirconia and solid materials plasma processing.

${ }^{19} \mathrm{R}$. D. Davis, The plasmachem. Process of producing refractory and refractory metal powders.

${ }^{20} \mathrm{P}$. H. Dundas, Plasma processing in Russia-A review.

${ }^{21}$ R. E. Gannon, Symposium on commercial potential for arc and plasma processes.

${ }^{22} \mathrm{P}$. Chung, Chemically reacting non-equilibrium boundary layers, Advances in Heat Transfer, Vol. 2, p. 109. Academic Press, New York (1965).

${ }^{23}$ S. Chapman and T. G. Cowling, The Mathematical Theory of Non-Uniform Gases. 2nd Edn., Cambridge University Press, Cambridge, Mass. (1952).

${ }^{24}$ R. C. Mason, Probe measurements on high pressure arcs, Phys. Rev. 51, 28 (1937).

${ }^{25}$ T. W. Petrie and E. Pfender, The effect of ionization on heat transfer to wires immersed in a highly thermally-ionized plasma, Wärme - und Stoffübertragung, 5 (2) 85 (1972).

${ }^{26}$ I. Kimura and A. Kanzawa, Experiments on heat transfer to wires in a partially ionized argon plasma, AIAA J. 3, 476 (1965).

${ }^{27} \mathrm{~A}$. Kanzawa, Heat transfer from a plasma to a solid wall with potential difference, Bull. Japan. Soc. Mech. Eng. 11, 664 (1968).

${ }^{28}$ T. N. Meyer and E. Pfender, Experimental and analytical aspects of plasma heat transfer, Wärme- und Stoffübertragung 6 (1) 25 (1973).

${ }^{29} \mathrm{~A}$. Kanzawa, Experiments of plasma heat transfer by thermocouple, Translated from Kagaku Kogaku 36, 1004 (1972).

${ }^{30} \mathrm{~A}$. Kanzawa and S. Nonouchi, Distributions of current density and heat flux around a cylindrical probe in an atmospheric pressure plasma, Translated from Kagaku Kogaku 38, 643 (1974).

${ }^{31} \mathrm{~T}$. N. Meyer, Effects of applied voltage and surface chemistry on the heat flux to a probe immersed in an arc plasma, Ph.D. Thesis, University of Minnesota (1971).

${ }^{32}$ J. A. Fay and N. H. Kemp, Theory of heat transfer to a shocktube-end-wall from an ionized gas, J. Fluid Mech. 21, 659 (1965).
${ }^{33} \mathrm{~T}$. W. Petrie, The effect of ionization on heat transfer to wires immersed in an arc plasma, Ph.D. Thesis, University of Minnesota (1969).

${ }^{34} \mathrm{~W}$. Finkelnburg and $\mathrm{H}$. Maecker, Elektrische Bögen und thermisches Plasma, Encyclopedia of Physics (ed. S. Flügge), Vol. 22, p. 254, Springer, Berlin (1956).

${ }^{35} \mathrm{G}$. Ecker, Electrode components of the arc discharge, Ergebnisse d. exakten Naturwiss. Vol. 33, p. 1, Springer, Berlin (1961).

${ }^{36}$ A. E. Guile, Arc-electrode phenomena, IEE Rev. 118, 1131 (1971).

${ }^{37} \mathrm{C}$. W. Kimblin, Anode phenomena in vacuum and atmospheric pressure arcs, IEEE Trans. Plasma Sci. PS-2 (4) 310 (1974).

${ }^{38}$ J. M. Sommerville, The Electric Arc. John Wiley, New York (1959).

${ }^{39} \mathrm{H}$. C. Ludwig, Current density and anode spot size in the gas tungsten arc, Welding Res. Suppl. 234 s (1968).

${ }^{40} \mathrm{~K}$. T. Shih, Anode current density in high current pulsed arcs, $J$. Appl. Phys. 43 (12), 5002 (1972).

${ }^{4} \mathrm{R}$. Beaudet and M. G. Drouet, Technique, applicable to circuit breakers for the measurement of the spatial distribution in an arc, IEEE Trans. Power Appl. Syst. 93 (4), 1054 (1974).

${ }^{42} \mathrm{~J}$. D. Cobine and E. E. Burger, Analysis of electrode phenomena in the high-current arc, J. Appl. Phys. 26 (7), 895 (1955).

${ }^{43} \mathrm{~K}$. T. Shih and R. Dethlefsen, Anode heat flux density of high current arcs, J. Heat Transfer 93 (Series C) 1, 119 (1971).

${ }^{44} \mathrm{R}$. Paulson and E. Pfender, Anode heat transfer in a DC electric arc with superimposed axial flow, IEEE Trans. Plasma Sci. PS-1 (3) 65 (1973).

${ }^{45} \mathrm{~J}$. Smith and E. Pfender, Determination of local anode heat fluxes in high intensity, thermal arcs, IEEE Trans. Power Apparatus Sys. PAS-95 (2), 704 (1976).

${ }^{46}$ A. Wutzke, E. Pfender and E. R. G. Eckert, Study of electric-arc behavior with superimposed flow, AIAA J. 5, 707 (1967).

${ }^{47}$ A. Wutzke, E. Pfender and E. R. G. Eckert, Symptomatic behavior of an electric arc with a superimposed flow, AIAA J. 6, 1474 (1968). 